\title{
A NOVEL SYNTHESIS OF SUBSTITUTED 4-HYDROXY- 1,8-NAPHTHYRIDINES
}

\author{
ATMAKURI NARENDER, E.LAXMINARAYANA M. THIRUMALA CHARY* \\ *Kakatiya Institute of Technology and Science, \\ Yerragattu Hillock, Hasanparthy Mandal, Warangal - 506015 A.P., INDIA.
}

(Received: March 19, 2009- Accepted: August 29, 2009)

\begin{abstract}
Michael addition of 2-aminopyridines with methylpropiolate to afforded the addition compound $1^{\mathrm{a}-\mathrm{g}}$, which on further cyclisation at higher temperature in dowtherm-A to yield cyclised product $2^{\mathrm{a}-\mathrm{g}}$, with respective yields depends on the nature of the substituents.

Key words: Propionoic acid methyl ester, 2-aminopyridine and 3-(Pyridin-2-ylamino)-acrylic acid methyl ester.

\section{INTRODUCTION}

Nitrogen containing heterocyclic compounds have extensive pharmaceutical applications and biologically activity. 1,8-Naphthyridines are one of the important class of such compounds as they have an excellent biological activity ${ }^{1}$, and their chemistry is recently reviewed ${ }^{1-3}$. As a step in this direction and in the continuation of our work on 1,8-Naphthyridines ${ }^{4-7}$, synthesis of the compounds depicted in the title is carried out. The general synthetic procedures used in the preparation of these compounds involve the Michael type addition ${ }^{8-12}$. Several substituted 4-hydroxy-1,8-naphthyridine and 4-Hydroxy-7-amino-1,8-naphthyridines have been prepared by condensation of substituted amino pyridines with methyl propiolates in methanol to give intermediates 3-(Pyridin-2-ylamino)-acrylic acid methyl ester derivatives, further these intermediates are cyclised in Dowtherm- A at higher temperature, to yield hydroxy naphthyridines.
\end{abstract}

\section{Reaction Scheme:}

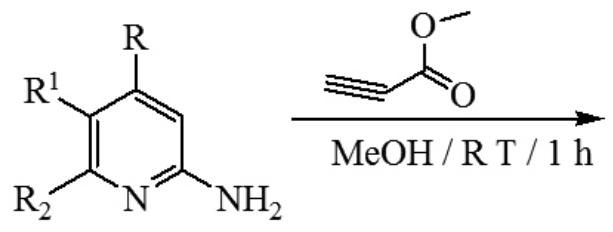

\section{EXPERIMENTAL}

Melting points were determined in open capillaries and are uncorrected. ${ }^{1} \mathrm{H}$ NMR spectrum is taken on a Varian $500 \mathrm{MHz}$ instrument with TMS as internal standard. The chemical shifts are expressed $\mathrm{d} \mathrm{ppm}$ and the solvent used is DMSO-d6. Mass spectrum is taken on Hewlett Packard mass spectrometer operating at $70 \mathrm{ev}$. All the compounds are recrystalised from ethanol.

General procedure for the preparation of compounds $\left(1^{\mathrm{a}-\mathrm{g}}\right)$ :

2-Amino pyridines $(10.60 \mathrm{mmol})$ is dissolved in $10 \mathrm{ml}$ of dry methanol and is added with Propiolicacid methyl ester $(16.0 \mathrm{mmol})$ drop wise at room temperature and stirred for about 16 hours. The methanol is evaporated completely under the reduced pressure. A reddish colour solids were obtained, The resulting reddish colour solids were recrystalised in ethyl acetate in hexane, yielded a light yellow coloured solids in very good yields.

General procedure for the preparation of 1,8-naphthyridines $\left(2^{a-g}\right)$ :

Compounds $\left(\mathbf{1}^{\mathrm{a}-\mathrm{g}}\right),(7.86 \mathrm{mmol})$ is taken in Dowtherm-A $(14 \mathrm{~mL})$ and refluxed about $250^{\circ} \mathrm{C}$ for $40-50$ minutes. The reaction mixture is cooled to $30^{\circ} \mathrm{C}$ and poured in hexane $(100 \mathrm{~mL})$. A black coloured solids is precipitated which is filtered and washed twice with hexane. The resulting solids is recrystalised from ethanol to offer title compounds as brown coloured solids in good yields.

1,8-Naphthyridin-4-ol $\left(2^{\mathrm{a}}\right)$ :

${ }^{1} \mathrm{H}_{\mathrm{NMR}}$ (DMSO-d, , 500MHz): 6.42 (S, 1H), 6.90-6.98 (t, 1H), 7.10-7.20 (d, 1H), 7.60-7.75 (t, 1H), 8.10-8.21 (d, 1H); ${ }^{13} \mathrm{C}-\mathrm{NMR}$ (500 MHz, DMSOd / TMS) $\delta: ~ 82.3,87.4(2 \mathrm{CH}), 128.2(1 \mathrm{CH}), 141.3(1 \mathrm{CH}), 156.4(1 \mathrm{CH}), 158.4$ $(1 \mathrm{CH}), 167.4(1 \mathrm{CH}), 177.4(1 \mathrm{CH})$; IR (KBr pellet): $3320(\mathrm{OH}), \mathrm{cm}^{-1}$; Mass $(\mathrm{m} / \mathrm{z}): 147[\mathrm{M}+1]$;Yield $74 \%$.

7-Amino-1,8-naphthyridin-4-ol ( $\left.2^{\text {b }}\right)$ :

${ }^{1} \mathrm{H}$ NMR (DMSO-d, 500MHz): $4.46\left(\mathrm{~S}, 2 \mathrm{H}, \mathrm{NH}_{2}\right), 5.24(\mathrm{~S}, 1 \mathrm{H}, \mathrm{OH})$, 6.80-6.95 (m, 2H), 7.63-7.76 (t, 1H), 8.90-8.99 (d, $1 \mathrm{H}) ;{ }^{13} \mathrm{C}-\mathrm{NMR}(500 \mathrm{MHz}$, DMSOd / TMS) $\delta: 89.3,92.3(2 \mathrm{CH}), 118.2(1 \mathrm{CH}), 142.3(1 \mathrm{CH}), 155.4(1 \mathrm{CH})$, $159.3(1 \mathrm{CH}), 165.4(1 \mathrm{CH})$; IR (KBr pellet): $3315(\mathrm{OH}), 2875\left(\mathrm{NH}_{2}\right), \mathrm{cm}^{-1}$; Mass (m/z): $162[\mathrm{M}+1]$; Yield $68 \%$.

5-Methyl-1,8-naphthyridin-4-ol $\left(2^{c}\right)$ :

${ }^{1} \mathrm{H}$ NMR (DMSO-d 6 , 500MHz): $2.21\left(\mathrm{~s}, 3 \mathrm{H}, \mathrm{CH}_{3}\right), 5.72(\mathrm{~s}, 1 \mathrm{H}, \mathrm{OH})$, 6.83-6.97 (m, 1H), 7.43-7.66 (t, $1 \mathrm{H}), 8.92-8.96(\mathrm{~d}, 1 \mathrm{H}) ;{ }^{13} \mathrm{C}-\mathrm{NMR}(500 \mathrm{MHz}$, $\left.\mathrm{DMSOd}_{6} / \mathrm{TMS}\right) \delta: 33.2,\left(\mathrm{CH}_{3}\right), 85.2,94.3(2 \mathrm{CH}), 128.2(1 \mathrm{CH}), 143.3(1 \mathrm{CH})$, $151.4(1 \mathrm{CH}), 156.3(1 \mathrm{CH}), 163.4(1 \mathrm{CH})$; IR $\left(\mathrm{KBr}\right.$ pellet): $3318(\mathrm{OH}), \mathrm{cm}^{-1}$; Mass (m/z): $162[\mathrm{M}+1]$; Yield $65 \%$.

6-Methyl-1,8-naphthyridin-4-ol $\left(2^{\mathrm{d}}\right)$ :

${ }^{1} \mathrm{H}$ NMR (DMSO-d $\left.\mathrm{d}_{6}, 500 \mathrm{MHz}\right): 2.25\left(\mathrm{~s}, 3 \mathrm{H}, \mathrm{CH}_{3}\right), 5.68(\mathrm{~s}, 1 \mathrm{H}, \mathrm{OH})$, 6.73-6.87 (m, 1H), 7.23-7.36 (t, 1H), 8.82-8.86 (d, $1 \mathrm{H}) ;{ }^{13} \mathrm{C}-\mathrm{NMR}(500 \mathrm{MHz}$, $\left.\mathrm{DMSOd}_{6} / \mathrm{TMS}\right) \delta: 34.2,\left(\mathrm{CH}_{3}\right), 84.2,92.3(2 \mathrm{CH}), 126.2(1 \mathrm{CH}), 133.3(1 \mathrm{CH})$, $153.4(1 \mathrm{CH}), 157.3(1 \mathrm{CH}), 166.4(1 \mathrm{CH})$; IR (KBr pellet): $3324(\mathrm{OH}), \mathrm{cm}^{-1}$; Mass (m/z): $162[\mathrm{M}+1]$;Yield $69 \%$

7-methyl-1,8-naphthyridin-4-ol ( $\left.2^{\mathrm{e}}\right)$ :

${ }^{1} \mathrm{H}$ NMR (DMSO-d, $\left.500 \mathrm{MHz}\right): 2.28\left(\mathrm{~s}, 3 \mathrm{H}, \mathrm{CH}_{3}\right), 5.64(\mathrm{~s}, 1 \mathrm{H}, \mathrm{OH})$ 6.78-6.92 (m, 1H), 7.25-7.38 (t, 1H), 8.52-8.66 (d, 1H); ${ }^{13} \mathrm{C}-\mathrm{NMR}(500 \mathrm{MHz}$, 
$\left.\mathrm{DMSOd}_{6} / \mathrm{TMS}\right) \delta: 36.4,\left(\mathrm{CH}_{3}\right), 83.2,91.3(2 \mathrm{CH}), 124.2(1 \mathrm{CH}), 132.3(1 \mathrm{CH})$ $152.4(1 \mathrm{CH}), 158.3(1 \mathrm{CH}), 164.4(1 \mathrm{CH})$; IR (KBr pellet): $3322(\mathrm{OH}), \mathrm{cm}^{-1}$; Mass (m/z): $162[\mathrm{M}+1]$;Yield $69 \%$.

6-Chloro-1,8-naphthyridin-4-ol ( $\left.2^{1}\right)$ :

$\left.{ }^{1} \mathrm{H} \mathrm{NMR} \mathrm{(DMSO-d}_{6}, 500 \mathrm{MHz}\right): 4.64$ (s, 1H, OH), 6.93 (m, 1H), 7.25-7.38 $(\mathrm{t}, 1 \mathrm{H}), 8.82-8.93(\mathrm{~d}, 1 \mathrm{H}) ;{ }^{13} \mathrm{C}-\mathrm{NMR}\left(500 \mathrm{MHz}, \mathrm{DMSOd}_{6} / \mathrm{TMS}\right) \delta: 36.4,\left(\mathrm{CH}_{3}\right)$ 83.2, $91.3(2 \mathrm{CH}), 124.2(1 \mathrm{CH}), 132.3(1 \mathrm{CH}), 152.4(1 \mathrm{CH}), 158.3(1 \mathrm{CH}), 164.4$ (1CH); IR (KBr pellet): $3318(\mathrm{OH}), \mathrm{cm}^{-1}$; Mass (m/z): $181[\mathrm{M}+1]$; Yield $75 \%$.

7-Methoxy-1,8-naphthyridin-4-ol ( $\left.2^{\mathrm{g}}\right)$ :

${ }^{1} \mathrm{H}$ NMR (DMSO-d $\left.{ }_{6}, 500 \mathrm{MHz}\right): 4.18\left(\mathrm{~s}, 3 \mathrm{H}, \mathrm{OCH}_{3}\right), 6.17$ (s 1H). 7.02-7.17 (d 1H), $8.33(\mathrm{~s} 1 \mathrm{H}), 8.89(\mathrm{~d} 1 \mathrm{H}), 9.51(\mathrm{~s} 1 \mathrm{H}) ;{ }^{13} \mathrm{C}-\mathrm{NMR}\left(500 \mathrm{MHz}, \mathrm{DMSOd}_{6}\right.$ / TMS) $\delta: 61.4,\left(\mathrm{OCH}_{3}\right), 81.2,94.3(2 \mathrm{CH}), 121.2(1 \mathrm{CH}), 137.3(1 \mathrm{CH}), 155.4$ $(1 \mathrm{CH}), 159.3(1 \mathrm{CH}), 163.4(1 \mathrm{CH}) ; 177$; Mass $(\mathrm{m} / \mathrm{z})$ : $\left(\mathrm{M}^{+}+1\right)$; Yield $71 \%$.

Characterization table of 4-Hydroxy 1,8-naphthyridines $2^{\mathrm{a}-\mathrm{g}}$

\begin{tabular}{|c|c|c|c|c|c|c|c|c|}
\hline \multirow{2}{*}{ Compd } & \multirow{2}{*}{$\mathbf{R}^{1}$} & \multirow{2}{*}{$\mathbf{R}^{2}$} & \multirow{2}{*}{$\mathbf{R}^{3}$} & $\begin{array}{c}\text { M. } \\
\text { Formula }\end{array}$ & $\begin{array}{c}\text { Melting } \\
\text { Points }\end{array}$ & $\mathbf{C}$ & $\mathbf{H}$ & $\mathbf{3}$ \\
\hline $2 \mathrm{a}$ & $\mathrm{H}$ & $\mathrm{H}$ & $\mathrm{H}$ & $\mathrm{C}_{8} \mathrm{H}_{6} \mathrm{~N}_{2} \mathrm{O}$ & $244-246$ & 65.75 & 4.14 & 19.17 \\
\hline $2 \mathrm{~b}$ & $\mathrm{H}$ & $\mathrm{H}$ & $\mathrm{NH}_{2}$ & $\mathrm{C}_{8} \mathrm{H}_{7} \mathrm{~N}_{3} \mathrm{O}$ & $262-264$ & 59.62 & 4.38 & 26.07 \\
\hline $2 \mathrm{c}$ & $\mathrm{Me}$ & $\mathrm{H}$ & $\mathrm{H}$ & $\mathrm{C}_{9} \mathrm{H}_{8} \mathrm{~N}_{2} \mathrm{O}$ & $225-227$ & 67.49 & 5.03 & 17.49 \\
\hline $2 \mathrm{~d}$ & $\mathrm{H}$ & $\mathrm{Me}$ & $\mathrm{H}$ & $\mathrm{C}_{9} \mathrm{H}_{8} \mathrm{~N}_{2} \mathrm{O}$ & $232-234$ & 67.49 & 5.03 & 17.49 \\
\hline $2 \mathrm{e}$ & $\mathrm{H}$ & $\mathrm{H}$ & $\mathrm{Me}$ & $\mathrm{C}_{9} \mathrm{H}_{8} \mathrm{~N}_{2} \mathrm{O}$ & $246-248$ & 67.49 & 5.03 & 17.49 \\
\hline $2 \mathrm{f}$ & $\mathrm{H}$ & $\mathrm{Cl}$ & $\mathrm{H}$ & $\mathrm{C}_{8} \mathrm{H}_{5} \mathrm{ClN}_{2} \mathrm{O}$ & $272-274$ & 53.21 & 2.79 & 15.51 \\
\hline $2 \mathrm{~g}$ & $\mathrm{H}$ & $\mathrm{H}$ & $\mathrm{OMe}$ & $\mathrm{C}_{9} \mathrm{H}_{8} \mathrm{~N}_{2} \mathrm{O}_{2}$ & $278-280$ & 61.36 & 4.58 & 15.90 \\
\hline
\end{tabular}

\section{CONCLUSIONS}

We have developed an economical, efficient cyclisation of amino pyridines with propioloic acid methyl ester, which can be a viable alternative to the Friedlander condensation. In the synthesis of all the derivatives, the same reaction conditions are provided. By this cyclisation one can prepare many of the 1,8-naphthyridine derivatives, which are useful intermediates to carryout the research.

\section{ACKNOWLEDGEMENT}

Authors are thankful to the Principal, Kakatiya Institute of Technology and Science-Warangal for providing necessary laboratory facilities and the Director IICT-Hyderabad, for providing spectral data.

\section{REFERENCES}

1. Egawa H, Miyamido A, Nishimra Y, Okada H, Uno H, Matsumato J. $J$ Med. Chem, 27,1543,(1984).

2. Nezval J and Halocka J, Experientia, 23,1043,(1967).

3. Suzuki N and R. Dohmori, Chem. Pharma. Bull, 27, 410,(1979).

4. Thirumala Chary M, Mogilaiah K and Sreenivasulu B. J. Indian Chem. Soc, 64,488,(1987).

5. Mogilaiah K, Vijayender Reddy K and Sreenivasulu B.Indian J. Chem, Sect.B, 22,178(1983).

6. Rao G R, Mogilaiah K and Sreenivasulu B, Collect Czech. Chem. Commun, 54,254,(1989).

7. Chary M T, Mogilaiah K, Swamy B and Sreenivasulu B, Sulfur Lett,

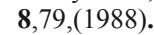

8. Vadim A Soloshonok, Chaozhong Cai, Takeshi Yamada, Hisanori Ueki, Yasufumi Ohfune, and Victor J. Hruby J. Am. Chem. Soc, 43,127,(2005).

9. Reddy K R, Kumar N S, Synlett, 6,224,(2006)

10. Wu F, Li L, Hong R and Deng L, Angew. Chem. Int. Ed, 45,947-950,(2006).

11. D. Bensa, T. Constantieux, J. Rodriguez, Synthesis, 36,923-927,(2004).

12. Ooi T, Ohara D, Fukumoto K, Maruoka K, Org. Lett, 7, 3195,(2005). 